\title{
Effects of Plasma Spatial Profile on Conversion Efficiency of Laser Produced Plasma Sources for EUV Lithography
}

\author{
A. Hassanein, V. Sizyuk, T. Sizyuk, and S. Harilal \\ Purdue University, West Lafayette, IN, USA
}

\begin{abstract}
Extreme ultraviolet (EUV) lithography devices that use laser produced plasma (LPP), discharge produced plasma (DPP), and hybrid devices need to be optimized to achieve sufficient brightness with minimum debris generation to support the throughput requirements of High-Volume Manufacturing (HVM) lithography exposure tools with long lifetime. Source performance, debris mitigation, and reflector system are all critical to efficient EUV collection and component lifetime. Enhanced integrated models are continued to be developed using HEIGHTS computer package to simulate EUV emission at high power and debris generation and transport in multiple and colliding LPP. A new center for materials under extreme environments (CMUXE) is established to benchmark HEIGHTS models for various EUV related issues. The models being developed and enhanced include, for example, new ideas and parameters of multiple laser beams in different geometrical configurations and with different pre-pulses to maximize EUV production. Recent experimental and theoretical work show large influence of the hydrodynamic processes on EUV generation. The effect of plasma hydrodynamics evolution on the EUV radiation generation was analyzed for planar and spherical geometry of a tin target in LPP devices. The higher efficiency of planar target in comparison to the spherical geometry was explained with better hydrodynamic containment of the heated plasma. This is not the case if the plasma is slightly overheated. Recent experimental results of the conversion efficiency (CE) of LPP are in good agreement with HEIGHTS simulation.
\end{abstract}

Keywords: EUV lithography, LPP, DPP, photon transport, MHD, HEIGHTS, CMUXE.

\section{INTRODUCTION}

Laser-produced plasma (LPP) devices are recently becoming more popular as sources of EUV production for the advanced lithography. Spitzer et al. (1996) [1] performed experimental study of laser irradiation on several materials for possible EUV radiation and showed results that tin is one of the most promised target material for EUV production around $13.5 \mathrm{~nm}$ wavelength. The experiment also examined the dependence of the conversion efficiency (CE) on the intensity of laser pulse, wavelength, and the pulse width of the laser beam.

In recent years, LPP processes for the optimization of EUV production were studied using several target materials such as xenon, lithium, and tin [2-4], different target geometry and composition (solid, liquid, cluster grains, etc) [5, 6], and various laser radiation parameters (radiation power density, wavelength, and pulse duration) [7]. Numerous experiments and computer simulations were made to increase the EUV radiation power. Such analysis also included the use of pre-pulses for formation of optimum plasma plume for EUV production during the main laser pulse [8-10]. The optimization of target geometry for efficient laser energy absorption was analyzed using liquid-jets [11], droplets with tuning of droplet size and laser spot size ratio [12], and the use of planar targets with various thicknesses [13]. The implementation of mass-limited Sn-doped targets for the production of optically thin plasma and minimization of tin debris was also studied [13,14]. The influence of spatial plasma effects using multi-laser systems for plasma confinement [15] and the uniformity of spherical target heating by multiple laser beams have indicated potential enhancement of EUV production [16]. Recent experimental and theoretical efforts $[9,17,18]$ show the importance of the hydrodynamic effects on the EUV generation process. These studies observed an increase in EUV emission near the target surface located close to the laser spot or in the location of the colliding plasma plumes. This effect is believed to be due to the geometrical confinement/containment of motion of the heated plasma, i.e., the density of the hot plasma increases in such locations with the optimum balance of the plasma density/temperature for the EUV generation.

* hassanein@purdue.edu; phone 1765 496-9731; fax 1765 496-2233

Metrology, Inspection, and Process Control for Microlithography XXIII, edited by John A. Allgair, Christopher J. Raymond Proc. of SPIE Vol. 7272, 72720K · C 2009 SPIE · CCC code: 0277-786X/09/\$18 · doi: 10.1117/12.813423 
Enhanced and integrated models are continued to be developed using HEIGHTS computer package to simulate EUV emission at high power and debris generation with mitigation/transport in multiple and colliding plasmas. A new center for materials under extreme environments (CMUXE) is established at Purdue University to benchmark HEIGHTS models against LPP designed experiments using various $\mathrm{Nd}$ :YAG and $\mathrm{CO}_{2}$ lasers. The models being developed and enhanced include, for example, new ideas and parameters of multiple laser beams in different geometrical configurations and with different pre-pulses to maximize EUV production. Another recent idea being pursued to enhance the production of EUV is to collide two plasma streams together. However, the study of the EUV generation in colliding plasma becomes more difficult because of the complex multi dimensional character of the hydrodynamic and radiation transport problem of the two moving plasma streams. Our objective in this study is to use the integrated HEIGHTS package to benchmark recent LPP experiments and to explore ways of maximizing EUV radiation power.

\section{MATHEMATICAL AND PHYSICAL MODEL}

In general, to construct comprehensive and efficient integrated models, the plasma formation and motion is solved using the set of four conservation laws: mass, momentum, energy, and magnetic field in two-temperature approximation [18]. The final hydrodynamic equation set for LPP devices in matrix form can be described in the following:

$$
\frac{\partial \mathbf{U}}{\partial t}+\frac{1}{r} \frac{\partial}{\partial r}[r \mathbf{F}(\mathbf{U})]+\frac{\partial \mathbf{P}(\mathbf{U})}{\partial r}+\frac{\partial \mathbf{G}(\mathbf{U})}{\partial z}=\mathbf{\Omega}
$$

where the hydrodynamic fluxes are

$$
\mathbf{U}=\left[\begin{array}{l}
\rho \\
\rho v^{r} \\
\rho v^{z} \\
e_{h} \\
e_{i}
\end{array}\right] \quad \mathbf{F}(\mathbf{U})=\left[\begin{array}{l}
\rho v^{r} \\
\rho v^{r} v^{r} \\
\rho v^{z} v^{r} \\
v^{r}\left(e_{h}+p_{h}\right) \\
v^{r}\left(e_{i}+p_{i}\right)
\end{array}\right] \quad \mathbf{P}(\mathbf{U})=\left[\begin{array}{l}
0 \\
p_{h} \\
0 \\
0 \\
0
\end{array}\right] \quad \mathbf{G}(\mathbf{U})=\left[\begin{array}{l}
\rho v^{z} \\
\rho v^{r} v^{z} \\
\rho v^{z} v^{z}+p_{h} \\
v^{z}\left(e_{h}+p_{h}\right) \\
v^{z}\left(e_{i}+p_{i}\right)
\end{array}\right]
$$

and the dissipative terms are combined in the source $\boldsymbol{\Omega}$ given as

$$
\boldsymbol{\Omega}=\left[\begin{array}{l}
0 \\
0 \\
0 \\
Q_{e, t h}+Q_{i, t h}+Q_{l a s}+Q_{r a d} \\
Q_{i, t h}+Q_{e i}
\end{array}\right]
$$

Here $Q_{e, t h}$ is the electron heat conduction, $Q_{i, t h}$ is the ion heat conduction, $Q_{r a d}$ is the radiation transport, and $Q_{e i}$ is the electron-ion interaction. We use Gaussian units unless indicated otherwise. The conservative form of the initial equations allows the use of the TVD method in the Lax-Friedrich formulation (TVD-LF) [19] for the solution of the convective stage given by Eq. (1). The numerical scheme for the TVD-LF method applied to the cylindrical symmetry case was described in Ref. [20]. Following the splitting method, the $Q$-terms in Eq. (3) are calculated separately with the second (dissipative) stage of the HEIGHTS solver and are used as correctors for the main TVD-LF solution [15]. An implicit numerical scheme with sparse matrix linear solvers is used for calculating the terms $Q_{e, t h}$ and $Q_{i, t h}$ [21, 22]. 
The electron-ion interaction term $Q_{e i}=3 \frac{m_{e} n_{e}}{m_{i} \tau_{e}}\left(k_{B} T_{e}-k_{B} T_{i}\right)$ is calculated for each hydrodynamic step and used as input in the right side of Eq. (1), where $m$ is the mass; $n_{e}$ and $\tau_{e}$ are electron concentration and the relaxation time, respectively; $e$ is the electron charge; and $k_{B}$ is the Boltzmann constant. Detailed description of HEIGHTS 3D Monte Carlo radiation transport model is given in Ref. [18].

Ideally, the laser absorption by a target should be treated in two phases: first by the cold, unperturbed solid/liquid target and then by the target having a plasma layer in front of the remaining solid/liquid target. Most of the hydrodynamics codes developed for infrared, visible, or UV lasers interaction consider the plasma creation as instantaneous, at least compared to the driving laser duration. In this approximation, the laser interacts only with the expanding plasma because the beam is reflected at the critical density, which is typically about a hundred times lower than the solid density. In reality, distinguished boundary does not exist between these two phases. This becomes important because of the complex hydrodynamic flows near the target surfaces where we should take into account the various energy input from laser radiation, i.e., absorption/reflection in solid/liquid target, absorption/reflection in target vapor, and absorption/reflection in plasma shape. Since the generation of a suitable initial EUV radiation area above the target surface consumes part of the laser beam energy and part of main pulse duration, the final efficiency of LPP device increases with decreasing the time needed for such preparation stage. This whole process includes gradual decreasing of laser absorption in cold material and increasing absorption in the hot plasma. It is also important to take into account the re-absorption of laser radiation in the evolving plasma after the reflection from the liquid target surface. For accurate modeling of the above described processes we used the available experimental optical properties for laser reflection from liquid tin $[23,24]$. The reflection coefficient can be derived from this data as:

$$
k_{r e f}=\frac{(n-1)^{2}+k^{2}}{(n+1)^{2}+k^{2}}
$$

where $k$ and $n$ are real and imaginary parts of the refractive index $\vec{n}=n+i k$. To model laser absorption in vapor material we used the approximation given in Ref. [25]. As it shown here, one of the main features of the collisioninduced absorption is a quadratic dependence of the absorption coefficient on the density (for our case this corresponds to the pair collisions) and a weak dependence on the temperature [26]. Based on this model, we calculated the absorption coefficient for the target vapor up to $0.7 \mathrm{eV}$ and fitted the dependence between the experimental data for the liquid target $[23,24]$ and the Bremsstrahlung coefficient given by [27]

$$
k_{a b s}^{l a s}=\frac{16 \pi Z n_{e}^{2} e^{6} \ln \Lambda(v)}{3 c v^{2}\left(2 \pi m_{e} k_{B} T_{e}\right)^{3 / 2}\left(1-v_{p}^{2} / v^{2}\right)^{1 / 2}}
$$

and to be scaled as:

$$
k_{a b s} \sim \rho^{2} T_{e}^{1 / 2}
$$

where $e, n_{e}, m_{e}, T_{e}$ are the electron charge, concentration, mass, and temperature, respectively; $Z$ is the normalized ionic charge; $v$ is the frequency of laser light; $v_{p}=\sqrt{\frac{n_{e} e^{2}}{\pi m_{e}}}$ is plasma frequency; and $k_{B}$ is the Boltzmann constant, and $\rho$ is plasma density. The Coulomb logarithm is given by 


$$
\ln \Lambda=\ln \left[\frac{3}{2} \sqrt{\frac{\left(k_{B} T_{e}\right)^{3}}{\pi n_{e}}} \frac{1}{Z e^{3}}\right] .
$$

\section{MODELING RESULTS AND DISCUSSION}

For studying the spatial hydrodynamic effects of spherical and planar targets on the EUV production we modeled laser interaction with both a tin droplet and a slab target. Similar laser parameters were used in both cases, i.e., $1.064 \mu \mathrm{m}$ laser wavelength with $70 \mu \mathrm{m}$ spot size and $8 \mathrm{~ns}$ (full width, half maximum) duration. The diameter of the droplet was chosen to be two times larger than the spot size to allow efficient utilization of the laser energy and the target can still be considered as spherical.

We compared the HEIGHTS integrated modeling with the experimental data obtained in our new CMUXE laboratory. Laser beam with $8 \mathrm{~ns}$ duration and $150 \mu \mathrm{m}$ spot size on planar target were used in both the experiment and for the HEIGHTS simulation. The dependence of the CE on laser beam intensity is shown in Fig.1 and demonstrates a good agreement with the models in both the magnitudes and the trends.

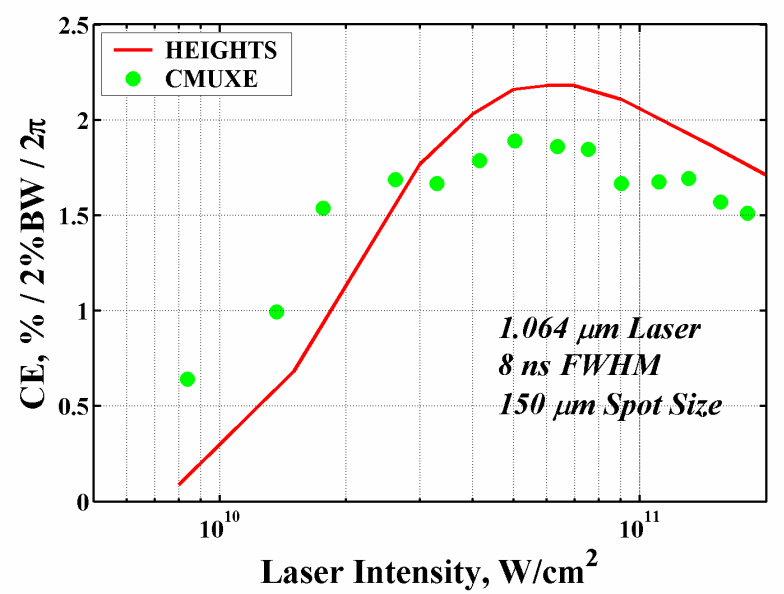

Fig.1 Dependence of CE on laser intensity both experimentally (CMUXE laboratory) and simulation (HEIGHTS package)

One of the main goals of this study is to determine the role of the hydrodynamic evolution and confinement processes as the main cause for the differences in the CE between the planar and spherical targets. HEIGHTS showed that planar targets provide greater geometrical plasma confinement in comparison to the spherical case. This hydrodynamic confinement prevents the plasma to quickly escape from the EUV production zone. Figure 2 shows the electrons temperature and density distributions at $4 \mathrm{~ns}$ after the peak of the laser beam with an intensity of $5 \times 10^{11} \mathrm{~W} / \mathrm{cm}^{2}$ (FWHM) irradiated the target surface. The target surface is located in all cases near the zero point. The plasma near target surface with electron density of around $10^{20} \mathrm{~cm}^{-3}$ and electron temperature of $\sim 30 \mathrm{eV}$ forms most productive EUV radiation area for these conditions. As shown in Fig. 3, significant part of the emitted photons is absorbed around this area in the denser zone (close to target surface) or in the more hot areas. The EUV radiation fluxes have larger values in surrounding regions.

HEIGHTS Monte Carlo model for radiation transport simulation permits to determine the location and intensity of photons source for EUV output in $2 \pi \mathrm{sr}$ (Fig. 4). The shape of EUV source area corresponds to the shape of the optimum plasma plume for photon production and depends on the area of the absorbing plasma. Figure 4 illustrates the source of EUV photons collected during 20 ns with laser pulse width 8 ns (FWHM). 

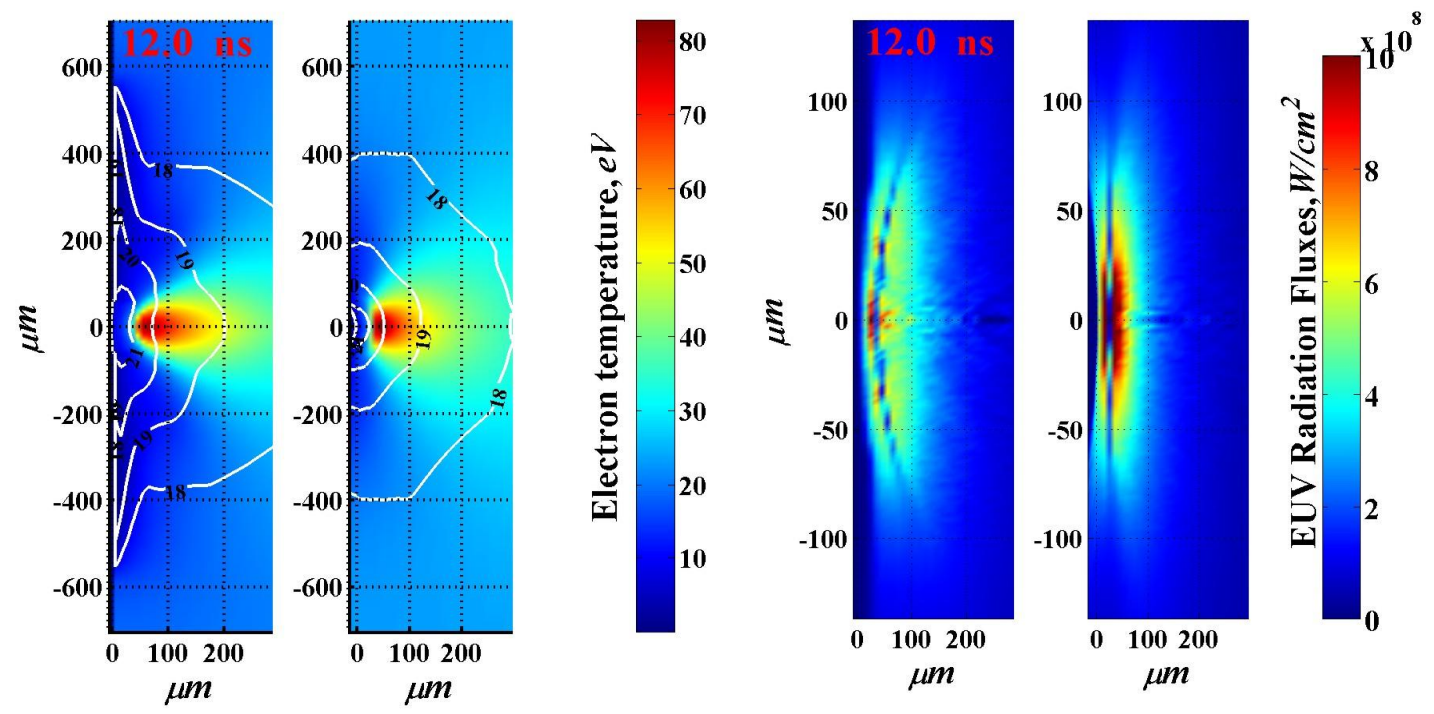

Fig.2 Electron temperature and density (white contours) distributions in planar (left) and droplet (right) target geometries
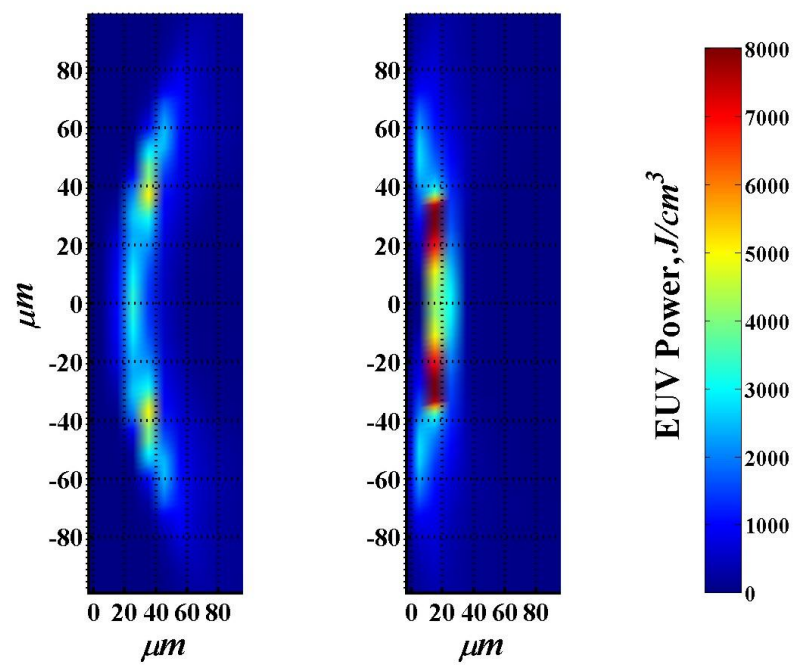

Fig.4 Location and intensity of EUV power collected during $20 \mathrm{~ns}$ in $2 \pi \mathrm{sr}$ in planar (left) and droplet (right) target geometries

As shown above, Figs. 2-4 correspond to an intense laser heating of $5 \times 10^{11} \mathrm{~W} / \mathrm{cm}^{2}$. This analysis shows that the effective plasma confinement in the case of high laser energy input causes plasma overheating and therefore, decreases the CE. Figure 5 confirms this observation. Planar target is more effective than droplet target for small and medium laser radiation intensities. The overheating reverses this situation for laser intensities higher than $\sim 2.5 \times 10^{11} \mathrm{~W} / \mathrm{cm}^{2}$.

At lower laser intensities the plasma is not excessively overheated and the size of EUV area is larger for the planar geometry because of better plasma evolution and containment. The EUV radiation area produced by laser heating of intensity $7 \times 10^{10} \mathrm{~W} / \mathrm{cm}^{2}$ is shown in Fig. 6. Most significant EUV photons generation zone is located around the spot 
center, in contrast to previously described regime. At higher laser intensities hydrodynamic plasma evolution moving away from the area of laser energy deposition/absorption prevents further plasma overheating in the system with the spherical configuration (Fig. 4). Hence, the difference in CE for planar and spherical target is explained by the nature of hydrodynamic geometrical containment. Condition with high plasma density and stable temperature should be created to get an optimum CE. The plasma temperature stability is provided by the balance between the incoming and escaping energy, i.e., laser energy absorption, radiation energy loss, and plasma expansion. The last term is mainly controlled by hydrodynamic confinement.

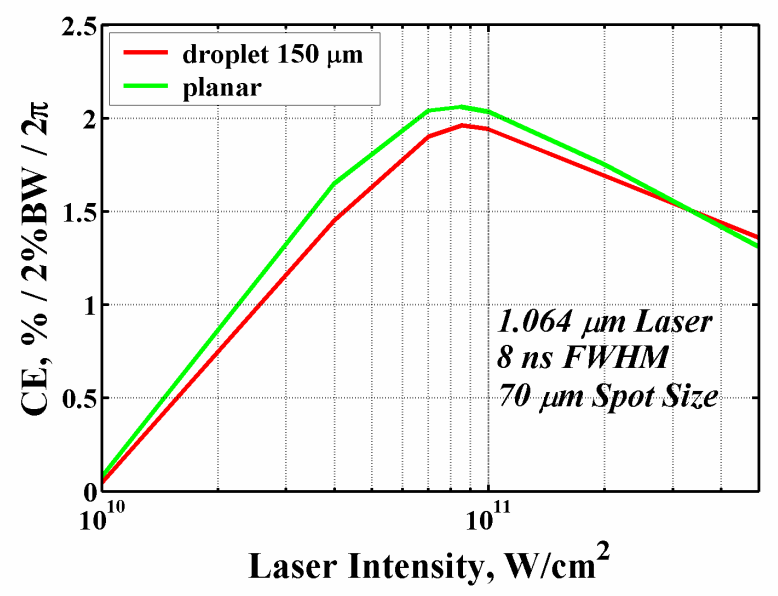

Fig.5 Comparison of laser intensity dependence of CE for sphere and planar geometries
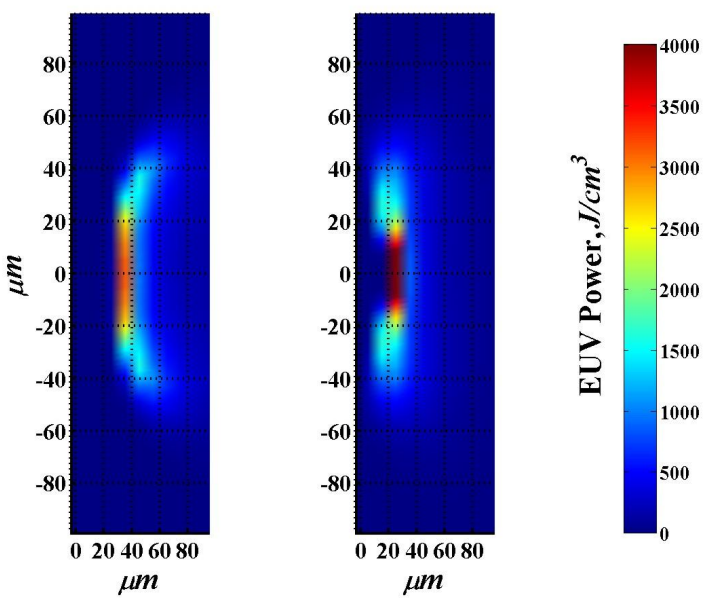

Fig.6 Location and intensity of EUV power collected during $20 \mathrm{~ns}$ in $2 \pi \mathrm{sr}$ in planar (left) and droplet (right) target geometries

The geometrical hydrodynamic effects control the plasma motion, i.e., limit and control the escaping plasma from the laser heating area and from the most productive EUV generation zone. In the case of the larger laser heating region, lower laser intensities are needed to create the efficient EUV production area because of the longer time of the plasma motion under the laser beam. The smaller laser irradiation spots produce opposite effects. As a result, the hydrodynamic confinement believed to be the cause of the right shift of the maximum $\mathrm{CE}$ due to the decrease of the laser beam radius (see Fig. 7). This is a consequence of the hydrodynamic evolution where the plasma confinement can play an important role. The main effects to maximize EUV production is to create the right plasma density and temperature conditions with maximum size for the longest possible period of time.

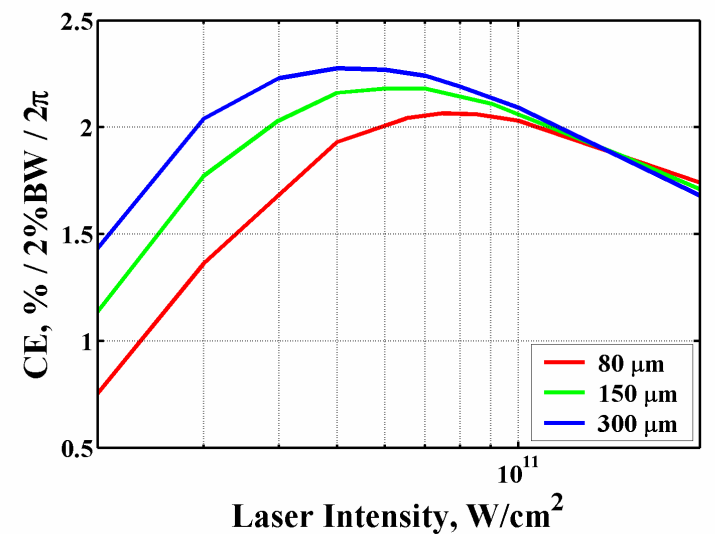

Fig.7 Laser intensity and spot size dependence of CE 


\section{CONCLUSION}

Enhanced integrated hydrodynamic, plasma, and photon transport models are continued to be developed and implemented in the HEIGHTS computer package to simulate EUV emission in both laser and discharge produced plasma devices. The models being developed and enhanced include, for example, detail spatial hydrodynamic plasma evolution, new ideas and parameters of multiple laser beams in different geometrical configurations, possible use of colliding plasma streams to enhance EUV production, and using combined laser sequences with different pre-pulses to maximize EUV production. A new center for materials under extreme environments (CMUXE) is established to benchmark HEIGHTS models for LPP source production and the study of debris effects on mirror reflectivity. Recent experimental results for LPP and the CE agree well with HEIGHTS models. The effect of plasma hydrodynamics evolution on the EUV radiation generation was further analyzed for planar and spherical geometry of a tin target in LPP devices. The higher efficiency of planar target in comparison to spherical geometry was explained with the better hydrodynamic geometrical containment of the heated plasma.

\section{REFERENCES}

1. Spitzer, R.C., Orzechowski, T.J., Phillion, D.W., Kauffman, R.L. and Cerjan, C., "Conversion efficiencies from laser-produced plasmas in the extreme ultraviolet regime", J.Appl.Phys. 79 (5) 2251-2258 (1996).

2. Shields, H., Fornaca, S.W., Petach, M.B., Michaelian, M., Mcgregor, D.R., Moyer, R.H., St. Pierre, R.J., " Xenon target performance characteristics for laser-produced plasma EUV sources," Proc. SPIE 4688, 94 (2002).

3. O'Sullivan, G., Cummings, A., Dunne, P., Hayden, P., McKinney, L., Murphy, N., and White, J., "Chapter 5: atomic physics of highly charged ions and the case for Sn as a source material," in EUV Source for Lithography, edited by V. Bakshi (SPIE, Bellingham, WA, USA 2006).

4. Nica, P.-E., Miyamoto, S., Amano, S., Inoue, T., Shimoura, A., Kaku, K., and Mochizuki, T., "Soft x-ray spectra from laser heated lithium targets," Appl. Phys. Lett., 89, 041501 (2006).

5. Abe, T., Suganuma, T., Imai, Y., Sugimoto, Y., Someya, H., Hoshino, H., Soumagne, G., Komori, H., Mizoguchi, H., Endo, A., and Toyoda, K., "Development of liquid-jet laser-produced plasma light source for EUV lithography," Proc. SPIE, 5037, 776 (2003).

6. Nagai, K., Gu, Q., Norimatsu, T., Fujioka, S., Nishimura, H., Miyanaga, N., Nishihara, K., Izawa, Y., and Mima, K., "Nano-structured lithium-tin plane fabrication for laser produced plasma and extreme ultraviolet generation," Laser Part. Beams, 26, 497 (2008).

7. Nishikawa, T., Sunahara, A., Sasaki, A., and Nishihara, K., "EUV Source Design Flexibility for Lithography," J. Phys.: Conf. Ser., 112, 042065 (2008).

8. Dusterer, S., Schwoerer, H., Ziegler, W., Salzmann, D., Sauerbrey, R., "Effects of a prepulse on laser-induced EUV radiation conversion efficiency", Appl.Phys.B 76, 17-21 (2003).

9. de Bruijn, R., Koshelev, K.N., Zakharov, S.V., Novikov, V.G., Bijkerk, F., "Enhancement of laser plasma extreme ultraviolet emission by shockwave-plasma interaction," Phys. Plasma 12, 042701 (2005).

10. Hoffman, L.R., Bykanov, A.N., Khodykin, O.V., Ershov, A.I., Bowering, N.R., Fomenkov, I.V., Partlo, W.N., Myers, D.W., "LPP EUV Conversion Efficiency Optimization", Proc. SPIE 5751, 892 (2005).

11. Hansson, B. A. M. and Hertz, H. M., "Liquid-jet laser-plasma extreme ultraviolet sources: from droplets to filaments", J. Phys. D: Appl. Phys., 37, 3233-3243 (2004).

12. Yuspeh, S., Sequoia, K.L., Tao, Y., Tillack, M.S., Burdt, R., and Najmabadi, F., "Optimization of the size ratio of Sn sphere and laser focal spot for an extreme ultraviolet light source", Appl. Phys. Lett., 93, 221503 (2008).

13. Fujioka, S., Nishimura, H., Okuno, T., Tao, Y., Ueda, N., Ando, T., Kurayama, H., Yasuda, Y., Uchida, S., Shimada, Y., Yamaura, M., Gu, Q., Nagai, K., Norimatsu, T., Furukawa, H., Sunahara, A., Kang, Y. -G., Murakami, M., Nishihara, K., Miyanaga, N., Izawa, Y., "Properties of EUV and particle generations from laserirradiated solid- and low-density tin targets", Proc. SPIE 5751, 578 (2005).

14. Hayden, P., Cummings, A., Murphy, N., O’Sullivan, G., Sheridan, P., White, J., and Dunne, P., "13.5 nm extreme ultraviolet emission from tin based laser produced plasma sources", J.Appl.Phys., 99, 093302 (2006).

15. Sizyuk, V., Hassanein, A., and Sizyuk, T., "Three-dimensional simulation of laser-produced plasma for extreme ultraviolet lithography applications", J. Appl. Phys., 100, 103106 (2006). 
16. Shimada, Y., Nishimura, H., Nakai, M., Hashimoto, K., Yamaura, M., Tao, Y., Shigemory, K., Okuno, T., Nishihara, K., Kawamura, T., Sunahara, A., Nishikawa, T., Sasaki, A., Nagai, K., Norimatsu, T., Fujioka, S., Uchida, S., Miyanaga, N., Izawa, Y., Yamanaka, C., "Characterization of extreme ultraviolet emission from laserproduced spherical tin plasma generated with multiple laser beams", Appl. Phys. Lett., 86, 051501 (2005).

17. Tillack, M.S., Sequoia, K.L., and Tao, Y., "Geometric effects on EUV emissions in spherical and planar targets," J. Phys.: Conf. Ser., 112, 042060 (2008).

18. Sizyuk, V., Hassanein, A., and Sizyuk, T., "Hollow laser self-confined plasma for extreme ultraviolet lithography and other applications", Laser Part. Beams, 25, 43, (2007).

19. Leveque, R.J., [Finite volume methods for hyperbolic problems], Cambridge: Cambridge University Press (2002).

20. Hassanein, A., Sizyuk, V., Tolkach, V., Morozov, V. \& Rice, B.J. "HEIGHTS initial simulation of discharge produced plasma hydrodynamics and radiation transport for extreme ultraviolet lithography," J. Microlith., Microfab., Microsyst., 3, 130 (2004).

21. Miloshevsky, G.V., Sizyuk, V.A., Partenskii, M.B., Hassanein, A., and Jordan, P.C., "Application of finitedifference methods to membrane-mediated protein interactions and to heat and magnetic field diffusion in plasmas," J. Comp. Phys., 212, 25 (2006).

22. Sizyuk, V., Hassanein, A., Morozov, V., Tolkach, V., Sizyuk, T., "Numerical simulation of laser-produced plasma devices for EUV lithography using the heights integrated model", Num. Heat Trans., A, 49, 215 (2006).

23. Petrakian, J.P., Cathers, A.R., Parks, J.E., MacRae, R.A., Callcott, T.A., and Arakawa, E.T., "Optical properties of liquid tin between 0.62 and 3.7 eV", Phys. Rev. B, 21, 3043 (1980).

24. Cisneros, G., Helman, J.S., Wagner, C.N.J., "Dielectric function of liquid tin between 250 and $1100{ }^{\circ}$ C", Phys. Rev. B, 25, 4248 (1982).

25. Grigoryan, G.G., Leonov, A.G., Manykin, E.A., Rudenko, A.A., Sitnikov, M.G., and Starostin, A.N., "The near infrared $(0.8-2.6 \mu \mathrm{m})$ absorption spectrum of a dense sodium vapor and possible mechanisms of the spectrum formation", JETP, 97, 678 (2003).

26. Frommhold, L., [Collisional-induced absorption in gases], Cambridge Univ. Press: Cambridge, (1993).

27. Johnston, T.W., and Dawson, J.M., "Correct values for high-frequency power absorption by inverse bremsstrahlung in plasmas", Phys. Fluids 16, 722 (1973). 